\title{
Effects of color, pattern, and sound on the movement of houseflies
}

\author{
Han-Seul Ryu, Si-Yun Sung ${ }^{*}$, Chae-Won Lim, Jong-Uk Na \\ Korean Minjok Leadership Academy, Hoengseong, Korea
}

Email address:

stellasung95@gmail.com (Si-Yun Sung)

To cite this article:

Han-Seul Ryu, Si-Yun Sung, Chae-Won Lim, Jong-Uk Na. Effects of Color, Pattern, and Sound on the Movement of Houseflies. American Journal of BioScience. Vol. 2, No. 5, 2014, pp. 187-191. doi: 10.11648/j.ajbio.20140205.14

\begin{abstract}
As diseases spread by flies have continuously increased in frequency since the 1970s, methods to control the flies' motion have been studied. In this paper, a novel method is proposed by examining the impact of diverse sensory stimuli on houseflies' motion. Among various patterns and colors, red striped pattern was the best as fly repellent, and the pattern of white circles on a blue background the best attraction. For sound, a frequency of $10 \mathrm{kHz}$ and $20 \mathrm{kHz}$ had repelling power. When examined for its relationship with the alluring smell of food, red stripes repelled more than $26.3 \%$ of flies from the smell. Under the same conditions, $10 \mathrm{kHz}$ and $20 \mathrm{kHz}$ each showed repelling power over about $19 \%$ in the presence of an olfactory luring factor. Therefore, the pattern of red stripes and the auditory stimuli of $10 \mathrm{kHz}$ or $20 \mathrm{kHz}$ should be incorporated into various aspects of our lives, along with the living environment of disease-stricken areas.
\end{abstract}

Keywords: Auditory Stimuli, Fly Repellent, Housefly, ParticleTracker, Pest Control, Visual Stimuli

\section{Introduction}

Tropic diseases, such as trachoma, leishmaniasis, and sleeping disease, are spread about by flies. In the case of trachoma, a disease involving permanent scarring of the eye, over 500 million worldwide are suffering from the disease, of which 6 million people have become blind. Thus, preventing the contact of flies is a crucial task. Concerning this task, research regarding the impact of visual stimuli on flies' movement is in its beginning stage. One research concluded that zebra stripes were a result of natural selection as they repelled tsetse flies [1]. A different group of scientists proved that the color blue is three times more attractive to flies than yellow [2]. In the case of sound, most research focuses on proving the attractive effect of flies' mating sound [3, 4]. However, the experiments mentioned above are not conducted under the influence of olfactory stimuli. For the repelling factors to provide effective pest control, it is necessary to prove the effectiveness of the repellents against olfactory attraction.

Thus, this research devises the optimum method to combat houseflies by studying the effect of sensory stimuli on their movement. After discovering the best repelling visual and auditory stimuli, the effect of the repelling stimuli was compared to the luring effect of olfactory attraction.

\section{Material and Methods}

\subsection{Reaction to Visual Stimuli}

Six strips of paper $\left(5 \times 15 \mathrm{~cm}^{2}\right.$; orange, yellow, red, green, blue, and black) with striped pattern were prepared in the shape of a fly trap and painted with fly glue liquid. The number of flies on each paper was observed after three days. The same was done with the wave pattern and two types of circled patterns- white circles with color background and colored circles with white background. Then, we compared the best repellent and the three best attractions to confirm the effect of the optimal repellent.

\subsection{Reaction to Auditory Stimuli}

Most ultrasonic insect repellent devices provide frequencies that vary over a wide range from $10 \mathrm{kHz}$ to 55 $\mathrm{kHz}$; however, their efficacy of them was not proved by official data. Thus, this experiment aimed to determine the best fly-repelling frequency by testing the effect of various frequencies from $10 \mathrm{kHz}$ to $50 \mathrm{kHz}$ at $10 \mathrm{kHz}$ intervals. The movement of flies in response to the frequencies was examined in an acrylic chamber $\left(30 \times 30 \times 150 \mathrm{~cm}^{3}\right)$ equipped with a small opening door in the middle. The sound wave was emitted from a function generator, its 
frequency checked with an oscilloscope. A set of speakers was connected to the function generator and placed at one end of the acrylic chamber. The movement was recorded for 1 minute before, during, and after the auditory stimuli. The experiment was repeated two times with a fresh group of houseflies each time. The data of the first experiment was collected using the program ParticleTracker of Image $\mathbf{J}$ provided by NIH. 300 frames were created from each one-minute video, and the program recorded the horizontal pixel number $(0 \sim 550)$ for each particle per frame. We assorted the acquired numbers into 11 groups in a histogram with an interval of 50 pixels. The data was also made into a chart by classifying 11 groups to three regions of the chamber: close to the sound, middle, and far from the sound. We constructed the second experimental data by first capturing scenes from one-minute videos every 5 seconds; next, we counted the number of flies according to their location among the three regions of the chamber mentioned above. We computed the mean value of the number of flies in each region during three minutes and compared the values of each area.

\subsection{Comparisons of the Effect of Sensory Stimuli}

The same chamber and sound emission devices were used. The first experiment was done to compare the strength of the luring effect of the olfactory stimuli and the repelling effect of the visual stimuli - using the best repelling pattern discovered through the first section of the research. A fruit was placed in the left end, and the left one-third of the chamber was covered with the repelling pattern. The video was taken before and after the chamber was covered with the visual stimuli. The second experiment compared the attracting effect of olfactory stimuli with the repelling effect of the auditory ones, which were the two best repelling frequencies from the second section of the research. The fruit and speaker were both placed in the left end. Lastly, we compared the auditory repelling effect with the visual repelling effect by placing the speaker at the left end and covering the right one-third of the chamber with the repelling pattern.

\section{Results}

\subsection{Reaction to Visual Stimuli}

\subsubsection{Reaction to Different Colored Patterns}

Regardless of the pattern, blue was the color that attracted the most numbers of flies, and the green second as shown in Table 1. For patterns, stripes were the least attractive pattern, while white circles with colored background and wave pattern the most attractive. The result of the experiments that focused on circle and wave pattern confirmed that flies like circular patterns as shown in Table 2 . The circle patterns allured about $29.59 \%$ of the flies in average, while plain white and plain blue colors, in average, $20.41 \%$. Set 1 , unlike the prior experiment, did not clearly confirm that flies prefer blue color when compared with the color white; yet, Set 2 explicitly showed that flies are attracted to the color blue. Plain blue lured more flies than plain white and blue wave patterns, more than black and white waves. Comparing the result of Set 2 with that of Set 1 , we ensured that circle is the most effective alluring pattern.

Table 1. Flies reaction to different colored pattern (\#: number, \%: percent)

\begin{tabular}{|c|c|c|c|c|c|c|c|c|c|c|}
\hline \multirow[t]{2}{*}{ Color } & \multicolumn{2}{|c|}{ Stripes } & \multicolumn{2}{|c|}{ Circles } & \multicolumn{2}{|c|}{$\begin{array}{l}\text { White circles, colored } \\
\text { background }\end{array}$} & \multicolumn{2}{|c|}{ Waves } & \multicolumn{2}{|c|}{ Total } \\
\hline & $\#$ & $\%$ & $\#$ & $\%$ & $\#$ & $\%$ & $\#$ & $\%$ & $\#$ & $\%$ \\
\hline Orange & 0 & 0.00 & 0 & 0.00 & 3 & 18.75 & 3 & 12.00 & 6 & 12.50 \\
\hline Yellow & 0 & 0.00 & 1 & 16.67 & 3 & 18.75 & 4 & 16.00 & 8 & 16.67 \\
\hline Red & 0 & 0.00 & 1 & 16.67 & 0 & 0.00 & 3 & 12.00 & 4 & 8.33 \\
\hline Green & 0 & 0.00 & 1 & 16.67 & 4 & 25.0 & 6 & 24.00 & 11 & 22.92 \\
\hline Blue & 1 & 100.0 & 2 & 33.33 & 4 & 25.0 & 6 & 24.00 & 13 & 27.08 \\
\hline Black & 0 & 0.00 & 1 & 16.67 & 2 & 12.5 & 3 & 12.00 & 6 & 12.50 \\
\hline
\end{tabular}

Table 2. Flies reaction to circle and wave pattern with variance

\begin{tabular}{llllll}
\hline Set 1 (circle pattern) & \multicolumn{5}{c}{ Set 2 (wave pattern) } \\
\hline Pattern & Number & Percent (\%) & Pattern & Number \\
\hline Plain blue & 8 & 16.33 & Plain blue & 7 & 33.33 \\
Plain white & 12 & 24.49 & Plain white & 2 & 9.53 \\
White circles, blue background & 17 & 34.69 & Blue-white waves & 7 & 33.33 \\
Blue circles & 12 & 24.49 & Black-white waves & 5 \\
\hline
\end{tabular}

\subsubsection{Comparison of Different Fly Luring Color-Pattern and Fly Repelling Color-Pattern}

We conducted this experiment to confirm the previous results. The result was consistent with the experiments made before. As Table 3 demonstrates, green plain, yellow circles with white background and white circles with blue background all attracted more flies than red stripe, while the pattern of white circle with blue background caught the most flies. We could verify once again the attractiveness of the pattern of white circle with blue background and the 
strong repelling effect of red stripes.

Table 3. Effect of fly-luring and fly-repelling colored patterns

\begin{tabular}{lllll}
\hline Set & Type & Number & Total & Percent (\%) \\
\hline \multirow{2}{*}{ Set 1 } & Red stripes & 49 & \multirow{2}{*}{118} & 41.53 \\
& Plain green & 69 & & 58.47 \\
Set 2 & Red stripes & 58 & \multirow{2}{*}{128} & 45.31 \\
& Yellow circle & 70 & & 54.69 \\
& Red stripes & 41 & & 34.45 \\
Set 3 & White circles, blue & 78 & 119 & 65.55 \\
& background & & & \\
\hline
\end{tabular}

\subsubsection{Real-Life Application}

The result shown in Table 4 demonstrates that fly traps with blue circles are $44 \%$ point more effective in attracting flies, compared to the existing fly traps, which they have no pattern - white plain. Trash-containing vinyl bag, cloth, and plastic wrap with red stripes showed about $70 \%$ point higher fly-repelling rate than those without red stripes as demonstrated in Table 5. Flies were highly concentrated at plain white or transparent materials we use daily - five times more than at red striped materials.

Table 4. Effect of fly traps with blue circles and commercial fly trap (\#: number, \%: percent)

\begin{tabular}{|c|c|c|c|c|c|c|c|c|}
\hline \multirow{2}{*}{ Trap } & \multicolumn{2}{|c|}{ Barbeque Area } & \multicolumn{2}{|c|}{ Cafeteria } & \multicolumn{2}{|c|}{ Apartment } & \multicolumn{2}{|c|}{ Total } \\
\hline & $\#$ & $\%$ & $\#$ & $\%$ & \# & $\%$ & $\#$ & $\%$ \\
\hline White circles, blue background & 10 & 66.66 & 10 & 76.92 & 8 & 72.72 & 28 & 71.79 \\
\hline Commercial fly trap & 5 & 33.33 & 3 & 23.08 & 3 & 27.27 & 11 & 28.21 \\
\hline Total & 15 & 100 & 13 & 100 & 11 & 100 & 39 & 100 \\
\hline
\end{tabular}

Table 5. Effect of red striped wrap and white/transparent wraps(\#: number, \%: percent)

\begin{tabular}{|c|c|c|c|c|c|c|c|c|}
\hline \multirow{2}{*}{ Wrap } & \multicolumn{2}{|c|}{ Plastic bag } & \multicolumn{2}{|c|}{ Cloth } & \multicolumn{2}{|c|}{ Plastic Wrap } & \multicolumn{2}{|c|}{ Total } \\
\hline & \# & $\%$ & $\#$ & $\%$ & $\#$ & $\%$ & $\#$ & $\%$ \\
\hline Red stripes & 3 & 23.08 & 0 & 0 & 4 & 18.18 & 7 & 15.22 \\
\hline White/transparent & 10 & 76.92 & 11 & 100 & 18 & 81.81 & 39 & 84.78 \\
\hline Total & 13 & 100 & 11 & 100 & 22 & 100 & 46 & 100 \\
\hline
\end{tabular}

\subsection{Reaction to Auditory Stimuli}

\subsubsection{Effect of Frequency of $10 \mathrm{kHz}$ on Flies'Movement}

In the first round of the experiment, the movement distribution of flies close to the speaker reduced about $65 \%$ during the stimulus as shown in Fig. 1(a). In the second round, we noticed that flies' population also decreased about $30.1 \%$ during the stimulus. On the other hand, population increased about $47 \%$ at the region far from the sound when the sound was on in the second round. Thus, we concluded that sound of frequency $10 \mathrm{kHz}$ has fly-repelling effect.
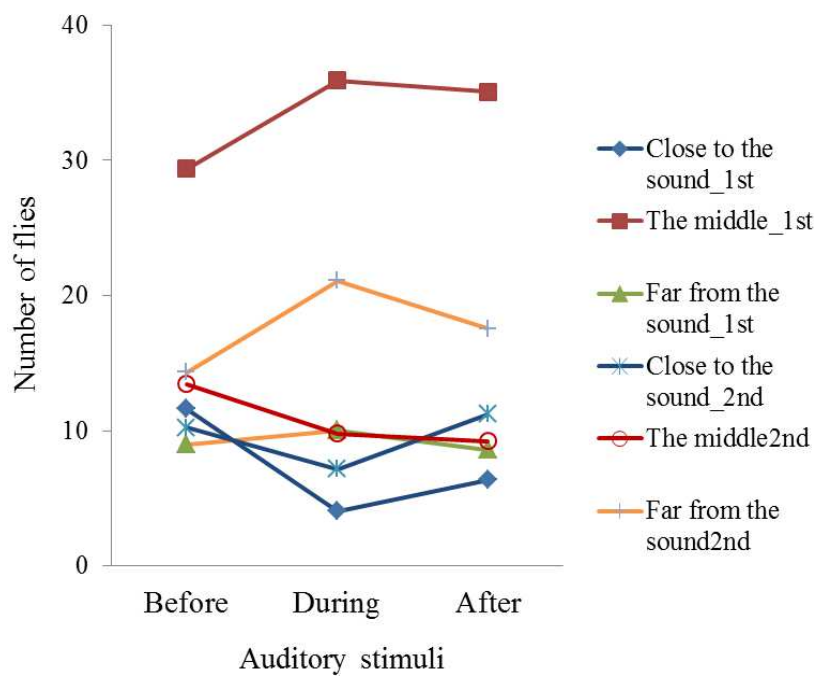

Auditory stimuli

\subsubsection{Effect of Frequency of $20 \mathrm{kHz}$ on Flies' Movement}

As shown in Fig. 1(b) the movement distribution in region close to the sound reduced in the first round when the sound was on. The number of flies closed to the sound reduced about $36.5 \%$ in the second round. However, when the sound was off, the movement distribution and the population of flies in the region close to the sound increased again. Thus, we concluded that $20 \mathrm{kHz}$ stimulus also has a repelling effect 


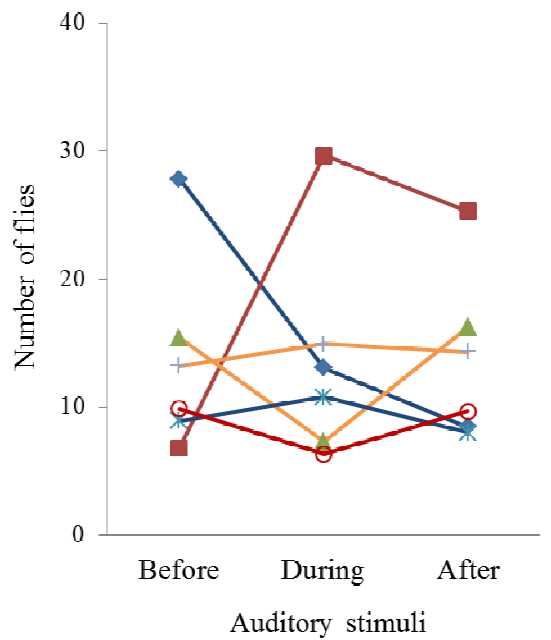

(c)

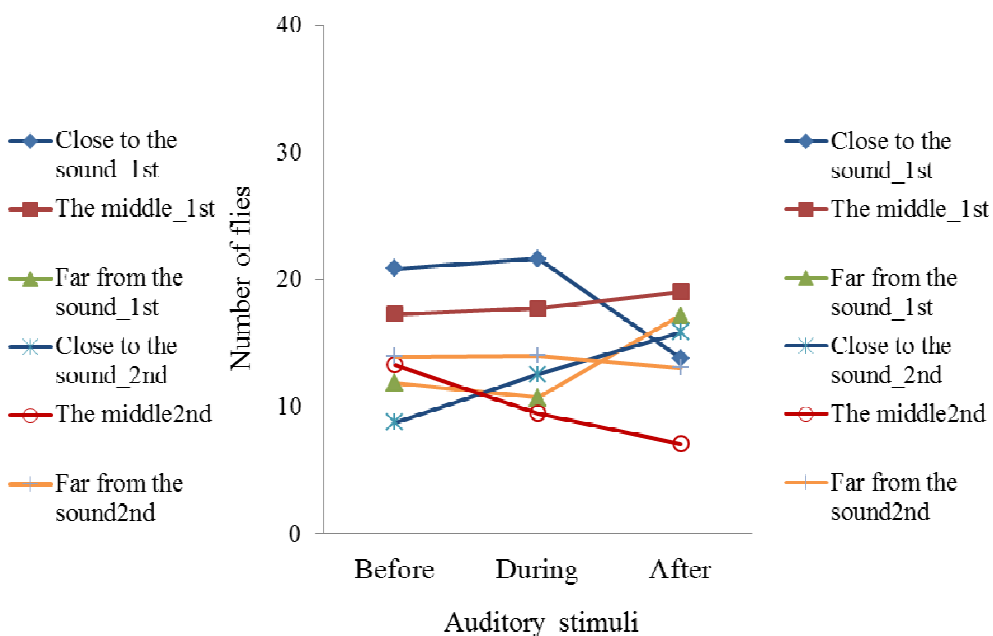

(d)

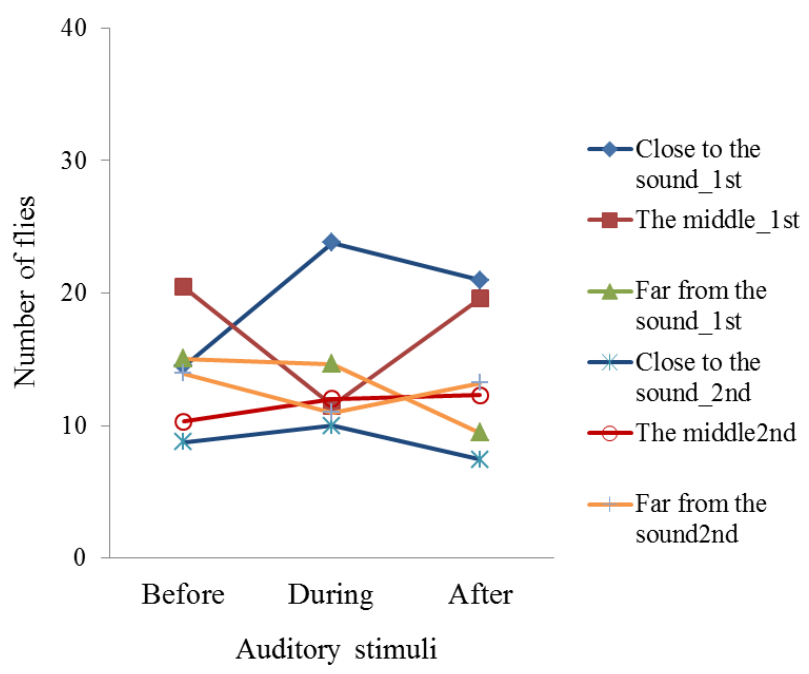

(e)

Figure 1. Reaction to auditory stimuli. (a) $10 \mathrm{kHz}$, (b) $20 \mathrm{kHz}$,(c) $30 \mathrm{kHz}$, (d) $40 \mathrm{kHz}$, (e) $50 \mathrm{kHz}$

\subsubsection{Effect of Frequency over $30 \mathrm{kHz}$ on Flies, \\ Movement}

Although there was distribution change when $30 \mathrm{kHz}$ and $40 \mathrm{kHz}$ stimuli was on, the tendency of flies' movement was not as explicit as it did during $10 \mathrm{kHz}$ and $20 \mathrm{kHz}$ stimuli. Therefore, we concluded that $30 \mathrm{kHz}$ and $40 \mathrm{kHz}$ stimuli gave no effect on flies' movement. $50 \mathrm{kHz}$ stimulus also did not provide impact on flies' movement, but the number of flies in the region closed to the sound had increased during the stimuli in both experiments.

\subsection{Comparisons of Diverse Sensory Stimuli}

\subsubsection{Comparison between the Effect of Olfactory and Visual Stimuli}

Before the visual stimulus, over $50 \%$ of fly population was near the olfactory stimulus. The effect of the olfactory stimulus was consistent even after the red striped paper (visual stimulus) was put around the chamber, as shown in
Table 6. However, the number of flies close to the food had decreased about $26.3 \%$ after the visual stimulus. This result demonstrates that the visual stimulus is able to repel a number of flies despite the olfactory stimulus.

Table 6. Average number of flies in each region of olfactory and visual stimuli

\begin{tabular}{lll}
\hline Region & Before visual stimulus & During visual stimulus \\
\hline Close to the food & 13.46 & 9.92 \\
The middle & 2.31 & 6.54 \\
Far from the food & 7.67 & 7.54 \\
\hline
\end{tabular}

\subsubsection{Comparison between the Effect of Olfactory and Auditory Stimuli}

The effect of olfactory stimulus was stronger on flies despite the auditory stimulus with repelling effect. However, $10 \mathrm{kHz}$ stimulus still affected flies movement, reducing over $31 \%$ of flies from region close to the food, and $20 \mathrm{kHz}$ stimulus reducing over $18.8 \%$ of flies as shown in Table 7 . 
Table 7. Average number of flies in each region of olfactory and auditory stimuli

\begin{tabular}{lllll}
\hline \multirow{2}{*}{ Region } & $\mathbf{1 0 ~ \mathbf { ~ H z }}$ & $\mathbf{2 0 k H z}$ & During auditory stimulus \\
\cline { 2 - 5 } & Before auditory stimulus & During auditory stimulus & Before auditory stimulus & 9.31 \\
\hline Close to the food & 16.15 & 11.15 & 11.46 & 5.46 \\
The middle & 6.7 & 7.69 & 7.08 & 9.23 \\
Far from the food & 6.15 & 10.16 & 5.46 & \\
\hline
\end{tabular}

\subsubsection{Comparison between the Effect of Visual and Auditory Stimuli}

Before the effect of $10 \mathrm{kHz}$ stimulus, $54.7 \%$ of flies population was concentrated in the region far from visual stimulus (the region closed to the sound), as shown in Table 8. Comparing the number of flies before and after $10 \mathrm{kHz}$ to that of during $10 \mathrm{kHz}$, we noticed that $10 \mathrm{kHz}$ stimulus reduced the effect of visual stimulus; the auditory stimulus still influenced flies despite the strong effect of visual stimulus. However, $46.2 \%$ of flies were still concentrated in the region far from the visual stimulus (close to the auditory stimulus), while only $24.3 \%$ of flies was in the region closed to the visual stimulus (far from the auditory stimulus). Thus, we concluded that the effect of visual stimulus is stronger in repelling flies than that of auditory stimulus of frequency $10 \mathrm{kHz}$; the same conclusion was made when we compared the repelling effect of visual stimulus and auditory stimulus of $20 \mathrm{kHz}$.

Table 8. Average number of flies in each region of visual and auditory stimuli

\begin{tabular}{llll}
\hline Region & Before 10khz & During 10khz & After 10khz \\
\hline Close to the sound & 24.62 & 20.77 & 23.62 \\
The middle & 12.54 & 13.31 & 11 \\
Far from the sound & 7.84 & 10.92 & 10.38 \\
\hline
\end{tabular}

\section{Discussion}

\subsection{Reaction to Visual Stimuli}

Red stripe pattern has the greatest repelling effect. This result is consistent with [5], which concluded that stripes of a zebra's coat reflect very different polarizations of light in a way that repel flies that reach zebras. Further, we found that the visual target with white circles in blue background has the strongest attractiveness. This is an advanced result of [2], which concluded that the color blue is the greatest attraction; they applied a pattern to the blue target, but it was limited to stripes, while we have tested diverse patterns and found the most effective.

\subsection{Reaction to Auditory Stimuli}

Frequency $10 \mathrm{kHz}$ and $20 \mathrm{kHz}$ has repelling effect on flies. According to [6], ultrasound induction had destroyed genomic DNA of housefly larvae; thus it can be considered that flies avoid $10 \mathrm{kHz}$ and $20 \mathrm{kHz}$ for reproductive reason.

\subsection{Comparisons of Diverse Sensory Stimuli}

The olfactory stimulus with luring effect is stronger than the effect of visual and auditory stimuli that repel flies. Nonetheless, red stripe and $10 \mathrm{kHz}$ to $20 \mathrm{kHz}$ stimuli reduced the luring effect of olfactory stimulus. Repelling effect of visual stimuli was stronger than that of the auditory stimuli.

\section{Conclusion}

Although olfactory stimulus had the strongest effect in luring flies, the visual stimulus with red stripes and auditory stimulus of $10 \mathrm{kHz}$ and $20 \mathrm{kHz}$ had repelling effect towards flies. Repelling effect of visual stimulus was stronger than the repelling effect of auditory stimulus. We recommend applying red stripes on various materials such as plastic bags, clothes, blankets, hats, etc. for pest control to get rid of germ-carrying flies and thus prevent possible diseases due to flies.

\section{References}

[1] Horváth, G., M. Blahó, G. Kriska, R. Hegedüs, B. Gerics, R. Farkas, and S. Åkesson. (2010). An unexpected advantage of whiteness in horses: the most horsefly-proof horse has a depolarizing white coat. Proceedings of the Royal Society B: Biological Sciences, 277(1688): 1643-1650.

[2] Diclaro, J.W., L.W. Cohnstaedt, R.M. Pereira, S.A. Allan and P.G. Koehler. (2012). Behavioral and physiological response of Musca domestica to colored visual targets. Journal of Medical Entomology, 49 (1): 94-100

[3] Webb, J.C., C.O. Calkins and D.L. Chambers, W. Schwienbacher, and K. Russ. (1983). Acoustical aspects of behavior of Mediterranean fruit fly, Ceratitis capitata: Analysis and identification of courtship sounds. Entomologia Experimentalis et Applicata, 33(1): 1-8.

[4] Mizrach, A,A. Hetzroni, M. Mazor, R. W. Mankin, T. Ignat, J. Grinshpun, N. D. Epsky, D. Shuman, R. R. Heath. (2005). Acoustic trap for female Mediterranean fruit flies. Transaction of the ASAE 4, 48(5): 2017-2022.

[5] Egri, Á.,M.Blahó, G. Kriska, R.Farkas, M. Gyurkovszky, S. Åkesson, andG. Horváth. (2012). Polarotactic tabanids find striped patterns with brightness and/or polarization modulation least attractive: an advantage of zebra stripes. The Journal of Experimental Biology, 215(5): 736-745.

[6] An, Chun-ju, F. Li, L.J. Zhang, D.S. Li, and R.Q. Du. (2005) Effects of ultrasound induction on genomic DNA of housefly larvae. GuangPu XueYu GuangPu Fen Xi, 25(12): 2020-2023. 